\title{
Manejo de estomas complicados y/o abdomen hostil con la técnica de condón de Rivera. Diez años de experiencia
}

\author{
Management of complicated stomas and/or hostile abdomen \\ with Rivera's condom technique. Ten years of experience \\ Miguel Ángel Rivera Pérez,* Bertha Karina Quezada González,**
Marlon Quiñónez Espinoza,*** Ramón René Almada Valenzuela****
}

Palabras clave: Abdomen hostil, estoma disfuncional, condón de Rivera.

Key words: Hostile abdomen, dysfunctional stoma, Rivera condom.

* Cirujano General encargado del Servicio de Abdomen Complicado y Nutrición Parenteral, Hospital General Regional Núm. 1. ** Cirujana General, adscrita al Hospital General Regional Núm. 1. *** Cirujano General, adscrito a la Unidad Médica de Alta Especialidad. **** Encargado de la Clínica de Heridas, Hospital General Regional Núm. 1.

Instituto Mexicano del Seguro Social (IMSS), Ciudad Obregón, Sonora.

Recibido: 17/05/2017 Aceptado: 12/10/2017

\section{RESUMEN}

\section{ABSTRACT}

Introducción: Se estima que la mitad de los pacientes con estoma intestinal van a tener complicaciones. En ocasiones, no se logrará un adecuado control de la fuga intestinal libre a la cavidad abdominal. Para estas situaciones, se diseñó una férula de látex al cabo distal intestinal, para exteriorizar el material intestinal y evitar la contaminación tanto química como bacteriana hacia la cavidad abdominal. Material y métodos: Se realizó una recolección prospectiva de pacientes con estoma complicado o algún grado de Björck que requirieron la utilización de férula de látex para el control de la fuga intestinal del 01 de agosto de 2006 al 31 de julio de 2016. Es un estudio de tipo descriptivo, prospectivo, longitudinal, de tipo cohortes. Resultados: Se incluyeron 658 pacientes; a 380 se les colocó condón y 278 fueron manejados con otros métodos. En quienes se colocó condón, la disminución de los valores de APACHE II fue de 24 a siete puntos. En relación con el índice de Mannheim, en los que se colocó el condón, los valores disminuyeron de 31 a 15 puntos. Conclusiones: Existen otros sistemas para el control de estomas; ninguno de ellos ha mostrado la versatilidad, facilidad de uso, practicidad y bajo costo de una férula con látex para el control del gasto intestinal, lo que muestra que es una técnica viable en todas las instituciones.
Introduction: It is estimated that half of the patients with intestinal stoma will have complications. In some cases, an adequate control of the free intestinal leakage to the abdominal cavity will not be achieved. For these situations, a latex splint on the distal end of the intestine was designed to externalize the intestinal material and avoid both chemical and bacterial contamination into the abdominal cavity. Material and methods: A prospective collection of made of all patients with a complicated stoma or some degree of Björck that required the use of latex splint for the control of intestinal leakage from August 1, 2006 to July 31, 2016. It is a descriptive, prospective, longitudinal, cohort-type study. Results: A total of 658 patients were included: 380 had condoms used on them and 278 were treated with other methods. In those in whom the condom was placed, the decrease in APACHE II values was from 24 to seven points. In relation to the Mannheim index, in those in whom the condom was placed, the values decreased from 31 to 15 points. Conclusions: There are other systems for the control of stomas; none of them has shown the versatility, ease of use, practicality and low cost of a latex splint for the control of intestinal expense, which shows that it is a viable technique in all institutions.

\section{INTRODUCCIÓN}

L a palabra estoma viene del griego $\sigma \tau o ́ \mu \alpha$ (boca) y médicamente se define como la apertura creada quirúrgicamente entre una víscera hueca y la superficie corporal u otra víscera. ${ }^{1}$
La interrupción de la continuidad del tubo digestivo está indicada en múltiples patologías abdominales: complicaciones infecciosas como perforaciones de víscera hueca, dehiscencia de anastomosis, necrosis segmentaria de una porción intestinal, entidades neoplásicas o casos de obstrucción intestinal. ${ }^{2}$ 
Las complicaciones de los estomas son frecuentes, afectan la calidad de vida y aumentan los costos para el sistema sanitario. Muchas están en relación con la técnica quirúrgica. $^{3}$

Se estima que la mitad de los pacientes con estoma intestinal van a tener complicaciones. No obstante, hay estudios que describen tasas del $70-80 \%$, o incluso del $96 \%$, durante las tres semanas siguientes a la cirugía. ${ }^{2-7}$ Este amplio rango dificulta precisar su incidencia debido a factores como duración del seguimiento, definición de las complicaciones, tipo de estoma, tipo de cirugía (urgente o diferida) o enfermedad de base. Dentro de las complicaciones tempranas se reportan cifras de hasta $82 \%$, donde las más comunes son irritación de la piel (55\%), problemas de fijación (46\%) y fuga $(40 \%){ }^{4}$

Todos estos reportes están enfocados en la formación de un adecuado estoma; sin embargo, ocasionalmente no es factible la adecuada realización del mismo debido al estado de sepsis y el nivel de hostilidad abdominal basado en adherencias intestinales, retracción mesentérica, retracción de aponeurosis, etcétera. Un número considerable de estos pacientes son consecuencia de un inadecuado manejo de abdomen abierto.

El método de abdomen abierto es una estrategia quirúrgica que consiste en el cierre diferido de la cavidad abdominal después de una laparotomía, como alternativa de manejo para los casos de sepsis intraabdominal, ya sea traumática o no traumática. ${ }^{7}$ El objetivo de este procedimiento es evitar la formación de focos sépticos múltiples y proteger la pared, disminuyendo el daño que presenta esta por cierres sucesivos. El abdomen abierto es una alternativa en la cirugía de control de daños y sepsis severa, pero la falta de sistematización del manejo y vigilancia estrecha del paciente podría aumentar la morbimortalidad y convertir un abdomen Björck I y II a un abdomen Björck III y IV. En pacientes con abdomen hostil, las perforaciones intestinales son frecuentes y son manejadas mediante cierre primario y/o ferulización con sondas; sin embargo, no en todos los casos se controla la fuga de material intestinal. La literatura no describe un método efectivo para control del gasto intestinal en estos pacientes.

La peritonitis generalizada es frecuentemente una condición letal. A finales del siglo XIX, la peritonitis era tratada médicamente, con una mortalidad de 90\%. En 1926, Krishner demostró que la mortalidad por peritonitis podía ser reducida con la implementación estricta de los principios quirúrgicos, y la tasa de mortalidad cayó por debajo del 50\%.

El pronóstico y desenlace de la peritonitis dependen de la interacción de varios factores, incluidos los relacionados con el paciente, la enfermedad, el equipo disponible, el nivel de hostilidad abdominal, la severidad de la infección, así como las intervenciones diagnósticas y terapéuticas. ${ }^{8}$

Varios sistemas de estadificación han sido utilizados para valorar el pronóstico y desenlace del paciente; entre ellos, APACHE II, índice de peritonitis de Mannheim, índice de peritonitis Altona, calificación de sepsis, y el POSSUM (Physiological and Operative Severity Score for Enumeration of Mortality and Morbidity). ${ }^{8}$

Meakins, Knaus y sus respectivos colaboradores reportaron que APACHE II es un mejor sistema pronóstico para el desenlace del paciente con peritonitis. ${ }^{9,10}$ Billing y su grupo concluyeron que el índice de peritonitis de Mannheim provee un medio más confiable para la evaluación del riesgo. ${ }^{11}$

El tratamiento de la sepsis abdominal es potencialmente quirúrgico, lo que controla la fuente de infección, remoción y drenaje de productos tóxicos, aliado a la antibioticoterapia, el soporte ventilatorio y la hemodinamia adecuada. $^{12}$

La cirugía de control de daños consta de tres pasos: 1) cirugía abreviada para un rápido control de la hemorragia y/o contaminación abdominal, con empaquetamiento hemostático y cierre abdominal temporal; 2) reanimación fisiológica; y 3) manejo quirúrgico definitivo. ${ }^{13}$

Algunos métodos utilizados en la cirugía de control de daños para control de origen del foco séptico son: realización de estomas, abdomen abierto con aseos quirúrgicos a demanda o programados, terapia de presión negativa. 
Es durante el proceso de reanimación y estabilización del paciente donde el método de exteriorización de la fístula por medio de un tubo de látex (colocación de un condón) resulta eficiente, ya que ayuda al control del foco séptico al prevenir que la fuga de contenido intestinal sea hacia la cavidad abdominal, lo que disminuye la irritación peritoneal y, por lo tanto, la respuesta inflamatoria sistémica.

\section{Tratamiento de las complicaciones precoces estomales}

Estas representan el $39-82 \%$ de las complicaciones de los estomas. ${ }^{2,3,4,14}$ Suponen un aumento de la estancia hospitalaria y los cuidados ambulatorios, lo que incrementa los costos tanto económicos como psicológicos. Estas complicaciones pueden ser graves, con necesidad de reintervenciones $(7 \%)$ y una mortalidad de $0.6-8 \%{ }^{3}$

Las complicaciones precoces pueden aparecer de forma aislada, pero se pueden asociar dos o más en el mismo paciente.

\section{Complicaciones de estomas}

Infección/absceso. Su tratamiento se realiza mediante drenaje y uso de antibioticoterapia, así como curaciones locales. En ocasiones, estos abscesos son secundarios a la presencia de fístulas: las superficiales cicatrizan espontáneamente, pero las persistentes y profundas requieren resección y reconstrucción del estoma con movilización del mismo para evitar la infección presente en el sitio primario. ${ }^{2,3,5}$

Dehiscencia de la unión mucocutánea. Puede ser limitada a un sector o al total de la unión, y es un factor predisponente de estenosis. ${ }^{2,3}$ Son esenciales los cuidados de enfermería para mantener limpio el espacio subcutáneo entre el estoma y la piel, rellenándolo con productos absorbentes, pasta o polvo, según la profundidad, hasta que la nueva unión mucocutánea se forme por segunda intención. ${ }^{3,5} \mathrm{En}$ casos más complejos se han empleado terapias de presión negativa. ${ }^{3}$

Necrosis. Es prioritario confirmar la extensión de la isquemia. Si la necrosis se extiende en profundidad bajo los planos miofasciales y peritoneo, está indicada la reoperación inmediata para resección y reconstrucción del estoma. $3,7,14,15$

Hundimiento. El hundimiento o retracción ocurre cuando el extremo del estoma se sitúa por debajo de $0.5 \mathrm{~cm}$ de la superficie de la piel durante las seis primeras semanas.

La retracción sin dehiscencia mucocutánea completa, con umbilicación del estoma, es lo más frecuente. En estos casos, se debe sellar el dispositivo sobre la pared abdominal para evitar el escape de heces, despegamiento de la bolsa y dermatitis irritativa. $2,3,4,16$

El hundimiento completo con desprendimiento mucocutáneo provoca contaminación subcutánea, pero si es subfascial, puede provocar peritonitis y sepsis, lo cual obliga a reintervenir de forma urgente. Si la mucosa es viable y no hay excesiva tensión, puede ser suficiente eliminar el tejido no viable, avanzar el asa intestinal y rehacer el estoma suturándolo a la piel; ;,16 sin embargo, habrá ocasiones en las que esto no sea técnicamente factible: es aquí donde se deben adoptar otras opciones de control del gasto intestinal para evitar la perpetuidad de estas complicaciones.

\section{Complicaciones cutáneas}

Son la causa de atención más habitual en la consulta por complicaciones de estoma. 1,4,14,15 Su incidencia es mayor en ileostomías, dado que las heces son más líquidas; las enzimas proteolíticas y el contenido alcalino pueden dañar la estructura epidérmica y provocar excoriación cutánea. ${ }^{14,16}$ El tratamiento consiste en limpieza cuidadosa de la piel con agua, secado y aplicación de pastas, polvos o cremas protectoras. ${ }^{5}$ Existe un arsenal de accesorios que han de adecuarse a las circunstancias de cada caso (polvos, pastas moldeables, discos convexos, cinturones, apósitos hidrocoloides absorbentes), ${ }^{3,5,7,16}$ pero a veces $-y$ sobre todo con el uso de placas convexas que ulceran por presión constante sobre la piel periestomal- se debe "descansar" de algún tipo de dispositivo sobre esa zona hasta su recuperación. Se han llegado a usar dispositivos tipo Penrose, pero se doblan y angulan, con lo que evitan el paso adecuado de la materia intestinal. 
Otra situación es la sepsis abdominal, donde el paciente se encuentra en tan malas condiciones que no es recomendable realizar una anastomosis intestinal, ${ }^{15}$ o cuando por el grosor del panículo adiposo es técnicamente imposible realizar un estoma de forma ordinaria. En esos casos, hay otras opciones de tratamiento, como realizar un estoma flotante (sin embargo, el control aún continuaría siendo difícil) mientras mejoran las condiciones del paciente para su cirugía definitiva.

\section{Estatificación de adherencias intestinales}

El grado de adherencias en la cavidad abdominal se puede determinar por la escala de Björck (Cuadro I), que las clasifica en cuatro grados dependiendo de sus condiciones. Para nuestro caso, podemos etapificar a los pacientes para ofrecerles una mejor opción de tratamiento sin arriesgar su integridad. ${ }^{17-19}$ Encontramos que en aquellos grupos que ya presentan, de acuerdo a esta escala, un grado 3 o (principalmente) 4 -que es un abdomen totalmente congelado- hay una nula posibilidad de realizar un estoma, y la mayoría

\section{Cuadro I.}

Clasificación de Björck 2009

\begin{tabular}{|c|c|c|c|}
\hline Grado & Descripción & Grado & Descripción \\
\hline $1 \mathrm{a}$ & Limpio sin adherencias & $1 \mathrm{a}$ & Limpio sin adherencias \\
\hline $1 b$ & Contaminado sin adherencias & $\begin{array}{l}1 \mathrm{~b} \\
1 \mathrm{c}\end{array}$ & $\begin{array}{l}\text { Contaminado sin adherencias } \\
\text { Líquido intestinal, asas } \\
\text { no fijas }\end{array}$ \\
\hline $2 \mathrm{a}$ & Limpio con adherencias fijas & $2 \mathrm{a}$ & Limpio con adherencias fijas \\
\hline $2 b$ & $\begin{array}{l}\text { Contaminado con } \\
\text { adherencias fijas }\end{array}$ & $\begin{array}{l}2 \mathrm{~b} \\
2 \mathrm{c}\end{array}$ & $\begin{array}{l}\text { Contaminado con } \\
\text { adherencias fijas } \\
\text { Líquido intestinal, asas fijas }\end{array}$ \\
\hline 3 & $\begin{array}{l}\text { Abdomen abierto } \\
\text { complicado, con fístula } \\
\text { en formación }\end{array}$ & $\begin{array}{l}3 a \\
3 b\end{array}$ & $\begin{array}{l}\text { Abdomen congelado limpio } \\
\text { Abdomen congelado } \\
\text { contaminado }\end{array}$ \\
\hline 4 & $\begin{array}{l}\text { Abdomen abierto } \\
\text { congelado, adherencias } \\
\text { firmes a intestino, imposible } \\
\text { de cerrar, con o sin fístula }\end{array}$ & 4 & $\begin{array}{l}\text { Abdomen congelado, fístula } \\
\text { entero atmosférica } \\
\text { establecida }\end{array}$ \\
\hline
\end{tabular}

de las veces no existe piel circundante para adaptar un dispositivo de recolección de material intestinal. Estos pacientes cursan con dermatitis química que lesiona cada vez más la piel perilesional. La terapéutica era dejarlos en ayuno y con nutrición parenteral por meses, lo que ocasionaba síndrome de sobrealimentación o complicaciones sépticas del catéter central en la mayoría de los casos. ${ }^{15,17,18}$

Al poder derivar el flujo intestinal fuera de la herida abdominal, se podría reiniciar la vía oral, estimulando la producción de sustancias que disminuyan la respuesta inflamatoria, aumentando el trofismo intestinal y llegando, incluso, a satisfacer la totalidad de sus requerimientos por vía enteral y a destetar de la nutrición parenteral. ${ }^{18,20,21}$

Al disminuir la irritación de la cavidad abdominal y la herida quirúrgica, se favorece la granulación y el cierre de la misma, a veces con la necesidad de rotación de colgajos o aplicación de auto- o xenoinjertos, para que ya con una cubierta cutánea, sea posible la aplicación de dispositivos convencionales para la recolección de flujos intestinales.

Algunos casos seleccionados se pueden manejar incluso como ambulatorios, con la manipulación de la dieta sin fibra, sin agua pura, solo electrolitos orales y la adición de loperamida o, en algunos casos de intestino ultracorto, el empleo de octreotide de larga duración. 7,18

\section{Control de complicaciones}

No existe, previo a esto, una técnica para el control de materia intestinal que logre ofrecer un manejo adecuado de la misma, sea fácil de usar y a bajo costo, para que se pueda utilizar en cualquier institución de segundo o tercer nivel de atención en el sector salud. Dependiendo de las alteraciones - locales o en toda la cavidad abdominal-, la labor del control de estoma se dificultará más. Ya que no se puede tener acceso a la cavidad ya congelada, el tratar de corregir el problema desde "abajo" causará fístulas y perforaciones a nivel abdominal. Para evitar fascitis o celulitis, se tiene que idear una técnica que logre cambiar el sitio de expulsión del material intestinal a otro 
donde no cause todo ese daño. Anteriormente, se ha reportado el uso de sondas urinarias para lograr este "control"; sin embargo, son expulsadas con la misma peristalsis intestinal y se obstruyen con las heces fecales. Otras alternativas, como tubos endotraqueales, no son más efectivas y pueden causar dolor en el paciente al tratar de deambular. Ante esta necesidad, se ideó la utilización de una férula del sitio donde se encontrase el cabo distal del estoma para que no existiera contaminación ni daño cercano a dicho sitio.

\section{Descripción de la técnica}

Lo primero es localizar el extremo proximal del intestino; dependiendo del estado de la mucosa, se tendrá o no la necesidad de infiltrarla, principalmente con lidocaína, para la eversión de la mucosa intestinal y, de esta manera, facilitar la sutura en los bordes de la misma. Para un mayor soporte de los líquidos intestinales, se utilizan preferentemente dos condones alineados, procurando que los dos anillos coincidan lo más posible para facilitar su sutura. Se aproxima el cabo proximal del condón al estoma (el lado

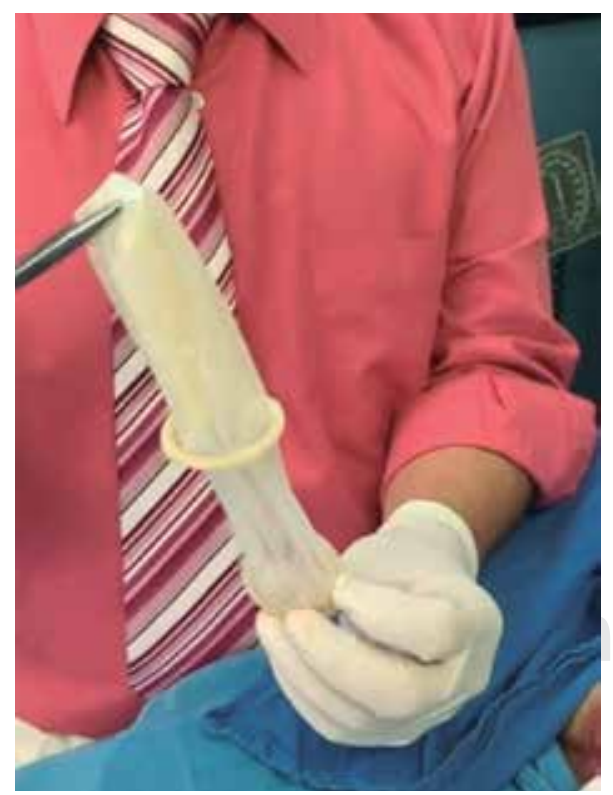

Figura 1. Expansión de los preservativos para su colocación. Archivo fotográfico del Dr. Rivera, con consentimiento informado.

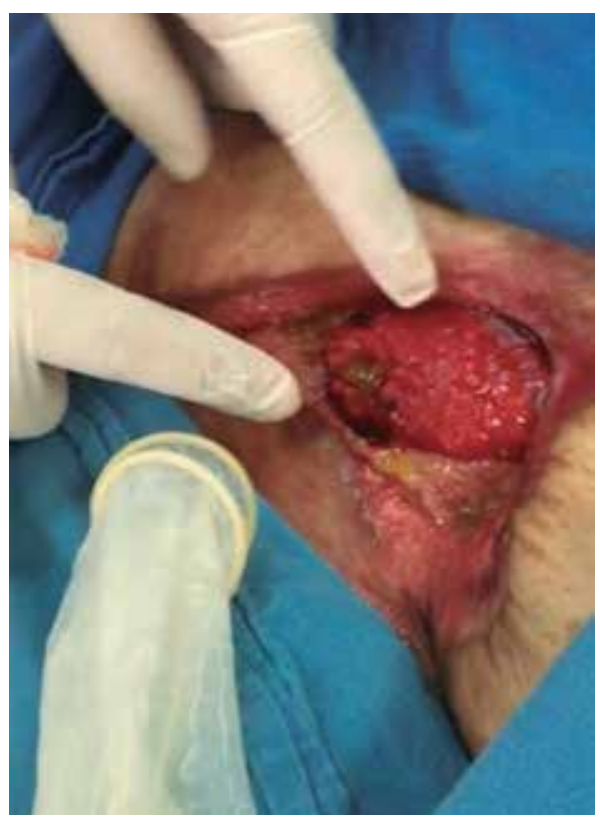

Figura 2. Visualización de la fístula y presentación de condón. Archivo fotográfico del Dr. Rivera, con consentimiento informado.

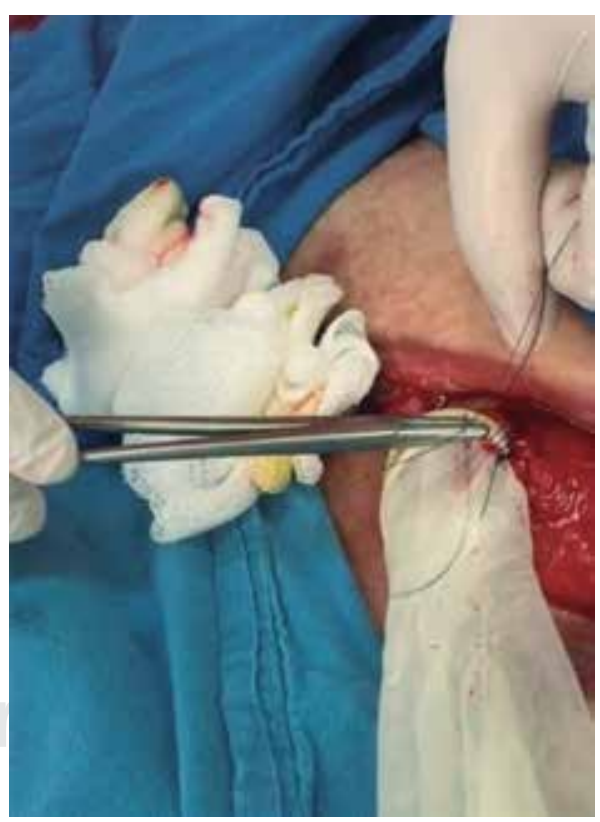

Figura 3. Se inicia sutura condón fístula, si se inicia al revés se desplaza el condón y es técnicamente más dificil. Archivo fotográfico del Dr. Rivera, con consentimiento informado. 


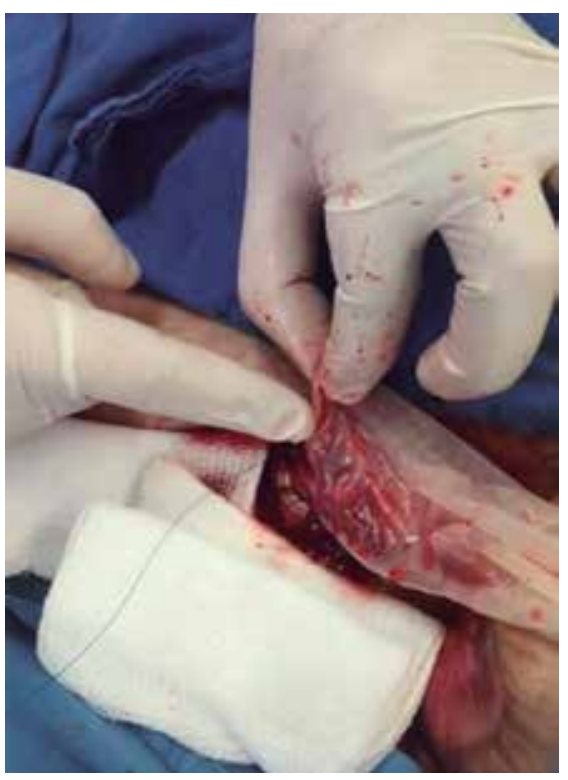

Figura 4. Se adapta el anillo del condón al diámetro de la fístula o perforación intestinal, si el anillo intestinal es más pequeño que el anillo del condón, el sobrante se sutura de manera vertical condón condón con sutura en greca, para evitar fugas. Archivo fotográfico del Dr. Rivera, con consentimiento informado.

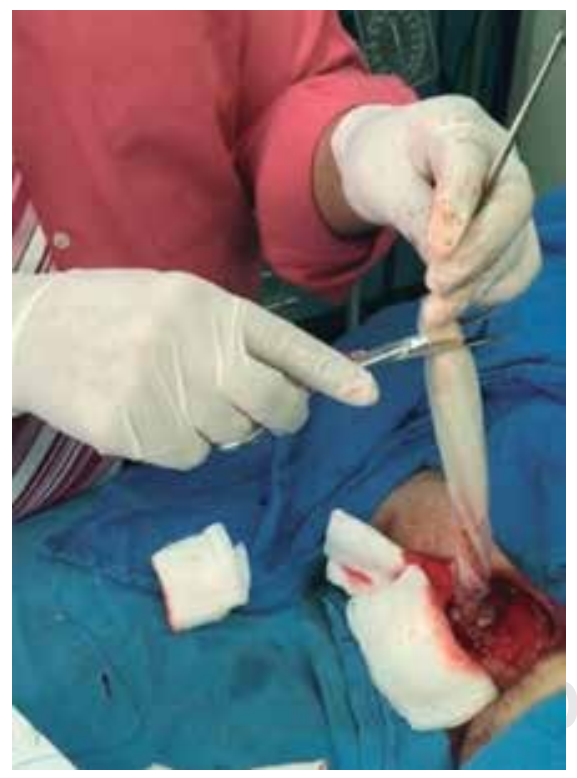

Figura 5. Se convierte el condón en un tubo de látex al cortar la punta del mismo, y de esta manera se forma un puente de salida del contenido intestinal fuera de la cavidad abdominal o de la herida quirúrgica. Archivo fotográfico del Dr. Rivera, con consentimiento informado.

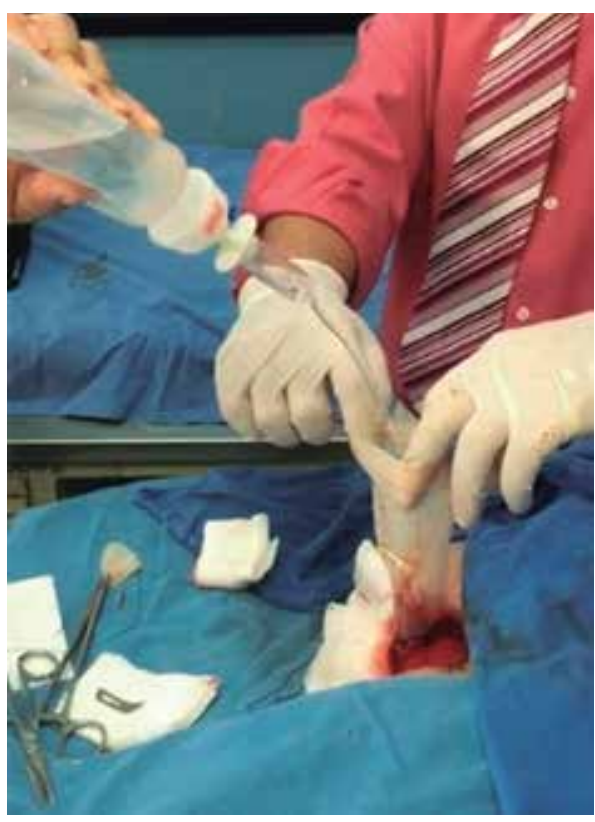

Figura 6. Se introduce agua o solución salina, para verificar hermeticidad y valorar la necesidad de reforzamiento en alguna área en especial. Archivo fotográfico del Dr. Rivera, con consentimiento informado.

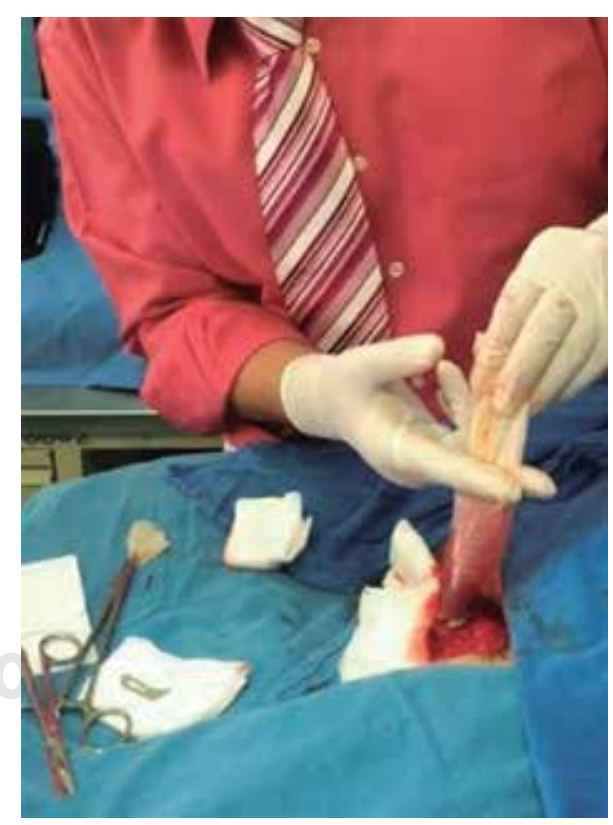

Figura 7. Se aplica presión hidrostática para verificar hasta la más mínima fuga. Archivo fotográfico del Dr. Rivera, con consentimiento informado. 
donde está el anillo), ya que este es el extremo con mayor firmeza, para evitar fugas periféricas en el área de sutura (Figura 1).

El intestino no tiene sensibilidad para dolor, solamente para frío, calor y tracción, por lo que no se requerirá algún tipo de anestesia especial para el procedimiento, sólo el uso de anestesia local en el tejido circundante si así lo amerita y su empleo para la eversión de bordes, como ya se comentó (Figura 2).

Para la anastomosis condón-intestinal, se utiliza de preferencia sutura monofilamento 2-0, con una técnica de surgete continuo, ya que un surgete anclado conferiría más isquemia local y tendería a la fuga prematura de la anastomosis; los puntos separados no confieren la adecuada hermeticidad y presentan igualmente fugas, no controlando el gasto intestinal (Figura 3).

En ocasiones, el estoma es más pequeño en diámetro que el diámetro de la base del condón; en estos casos, para evitar áreas de fuga, se realiza una sutura tipo greca en el área del condón sobrante. Si se intentara continuar con sutura continua, el área restante del con-

\section{Cuadro II. Escala Acute Physiology Chronic Health Evaluation o APACHE II.}

\begin{tabular}{|c|c|c|c|c|c|c|c|c|c|c|}
\hline \multicolumn{11}{|c|}{ Puntuación APACHE II } \\
\hline \multicolumn{2}{|l|}{ APS } & 4 & 3 & 2 & 1 & 0 & 1 & 2 & 3 & 4 \\
\hline \multicolumn{2}{|c|}{ Temperatura rectal $\left({ }^{\circ} \mathrm{C}\right)$} & $>40.9$ & $39-40.9$ & \multirow[b]{2}{*}{$110-129$} & $38.5-38.9$ & $36-38.4$ & $34-35.9$ & $32-33.9$ & $30-31.9$ & $<30$ \\
\hline \multicolumn{2}{|c|}{ Presión arterial media } & $>159$ & $130-159$ & & & $70-109$ & & 50.69 & & $<50$ \\
\hline \multicolumn{2}{|c|}{ Frecuencia cardiaca } & $>179$ & $140-179$ & $110-129$ & & 70-109 & & $55-69$ & $40-54$ & $<40$ \\
\hline \multicolumn{2}{|c|}{ Frecuencia respiratoria } & $>49$ & $35-49$ & & $25-34$ & $12-24$ & $10-11$ & $6-9$ & & $<6$ \\
\hline \multicolumn{11}{|c|}{ Oxigenación } \\
\hline \multirow{2}{*}{\multicolumn{2}{|c|}{$\begin{array}{l}\mathrm{Si} \mathrm{FiO} \\
2 \mathrm{Ci}_{2} \\
\mathrm{Si} \mathrm{FiO}_{2} \leq 0.5\left(\mathrm{AaO}_{2}\right)\end{array}$}} & $>499$ & $350-499$ & \multicolumn{2}{|l|}{$200-349$} & $<200$ & & & & \\
\hline & & & & & & $>70$ & $61-70$ & & $56-60$ & $<56$ \\
\hline \multicolumn{2}{|c|}{ pH arterial } & $>7.69$ & $7.60-7.69$ & \multirow[b]{2}{*}{$155-159$} & $7.50-7.59$ & 7.33-7.49 & & 7.25-7.32 & $7.15-7.24$ & $<7.15$ \\
\hline \multicolumn{2}{|c|}{ Na plasmático (mmol/l) } & $>1.79$ & $160-179$ & & $150-154$ & $130-149$ & & $120-129$ & $111-119$ & $<111$ \\
\hline \multicolumn{2}{|c|}{ K plasmático (mmol/l) } & $>6.9$ & $6.0-6.9$ & \multirow[b]{2}{*}{$1.5-1.9$} & $5.5-5.9$ & $3.5-5.4$ & $3.0-3.4$ & $2.5-2.9$ & & $<2.5$ \\
\hline \multicolumn{2}{|c|}{ Creatinina ${ }^{\circledR}(\mathrm{mg} / \mathrm{dl})$} & $>3.4$ & $2-3.4$ & & & $0.6-1.4$ & & $<0.6$ & & \\
\hline \multicolumn{2}{|c|}{ Hematocrito (\%) } & $>59.9$ & & $50-59.9$ & $46-49.9$ & $30-45.9$ & & $20-29.9$ & & $<20$ \\
\hline \multicolumn{2}{|c|}{ Leucocitos (x 1,000) } & $>39.9$ & & 20-39.9 & $15-19.9$ & $3-14.9$ & & $1-2.9$ & & $<1$ \\
\hline \multicolumn{11}{|c|}{ Suma de puntos APS } \\
\hline \multicolumn{11}{|l|}{ Total APS } \\
\hline Edad & Puntuación & \multicolumn{2}{|c|}{ Enfermedad crónica } & & $\begin{array}{l}\text { Puntos APS } \\
\text { (A) }\end{array}$ & $\begin{array}{l}\text { Puntos GCS } \\
\text { (B) }\end{array}$ & \multicolumn{2}{|c|}{$\begin{array}{l}\text { Puntos edad } \\
\text { (C) }\end{array}$} & \multicolumn{2}{|c|}{$\begin{array}{l}\text { Puntos enfermedad previa } \\
\text { (D) }\end{array}$} \\
\hline$\leq 44$ & 0 & \multirow{3}{*}{\multicolumn{2}{|c|}{$\begin{array}{l}\text { Postoperatorio } \\
\text { programado } \\
\text { Postoperatorio } \\
\text { urgente en médico }\end{array}$}} & 2 & \multicolumn{6}{|c|}{ Total de puntos APACHE II (A + B + C + D) } \\
\hline $45-54$ & 2 & & & 5 & Enfermedad cr & nica: & & & & \\
\hline $55-64$ & 3 & & & & $\begin{array}{l}\text { Hepáticas: cirro } \\
\text { Cardiovascular }\end{array}$ & $\begin{array}{l}\text { is (biopsia) o hi } \\
\text { disnea o angir }\end{array}$ & $\begin{array}{l}\text { ipertensión } \\
\text { na de repo }\end{array}$ & $\begin{array}{l}\text { ortal o episoc } \\
0 \text { (clave IV }\end{array}$ & $\begin{array}{l}\text { odio previo de fal } \\
\text { de NYHA) }\end{array}$ & hepático \\
\hline $65-74$ & 5 & & & & $\begin{array}{l}\text { Respiratoria: EF } \\
\text { Renal: diálisis }\end{array}$ & $\begin{array}{l}\text { OC grave, con } \\
\text { rónica }\end{array}$ & hipercapni & , policitemia & a o hipertensión & Ilmonar \\
\hline$\geq 75$ & 6 & & & & Inmunocompro & netidos: tratami & iento inmu & 1osupresor, in & inmunodeficienci & crónica \\
\hline
\end{tabular}

Fuente: Knaus WA, Draper EA, Wagner DP, Zimmerman JE. APACHE II: a severity of disease classification system. Crit Care Med. 1985; 13 (10): 818-829. 
dón presentaría ondulaciones que tenderían a fugar más; esta opción es la que ha mostrado mejor manejo y menor riesgo de fugas a través del condón (Figura 4).

El paso final es cortar la punta del condón. Se realiza una prueba neumática, que se logra introduciendo una pequeña cantidad de líquido dentro de la férula de látex; se ocluye el segmento distal y se lleva a cabo una taxis sobre el trayecto del condón, ejerciendo presión hacia la base del mismo para corroborar la ausencia de fugas alrededor de la anastomosis. Este último paso convertirá el condón en un tubo de látex; de esta manera, se puede canalizar todo el flujo intestinal hacia una bolsa de colostomía lejos de la herida quirúrgica complicada (Figuras 5 a 7).

\section{MATERIAL Y MÉTODOS}

Población de estudio:

Todo paciente con presencia de estoma complicado y abdomen hostil Björck 3 o 4, y que requiriera la utilización de férula de látex con técnica de condón de Rivera.

Se trató de un estudio de tipo descriptivo, prospectivo, longitudinal, de cohortes. Se incluyeron 658 pacientes con diagnóstico de sepsis abdominal secundaria a fístula intestinal del Hospital General Regional Núm. 1 del Centro Médico Nacional del Noroeste, en el periodo del 01 de agosto de 2006 al 31 de julio de 2016. Todos fueron intervenidos quirúrgicamente y se les exteriorizó una fístula por medio de un condón de látex; se tomaron valores de la escala APACHE II (Cuadro II) en al menos ocho días subsecuentes a la laparotomía inicial y se tomaron los valores del índice de Mannheim (Cuadro III) de tres laparotomías subsecuentes según los hallazgos quirúrgicos.

Se incluyeron en el estudio pacientes adultos con diagnóstico de sepsis abdominal secundaria a fuga intestinal, postoperados de laparotomía en el periodo del 01 de agosto de 2006 al 31 de julio de 2016.

Se excluyeron pacientes pediátricos (dado que el Servicio de Cirugía General no atiende pacientes menores de 18 años), aquellos con foco séptico sincrónico extra- abdominal (ya que la presencia de dicho foco infeccioso ocasionaría que los valores en la escala de APACHE II no disminuyeran en la forma esperada con la resolución del foco abdominal), los diagnosticados con infección por VIH/SIDA (dado que dicha patología interviene con el sistema inmune del paciente y ocasiona que su respuesta a la sepsis no sea la adecuada, lo que podría generar un sesgo en los resultados obtenidos), aquellos con sepsis abdominal que no fueron operados (ya que sin manejo quirúrgico, la obtención del índice de Mannheim no es posible), y los que estuvieran en tratamiento con esteroides (debido a que estos medicamentos alteran la cicatrización y respuesta inmune, lo que ocasionaría un sesgo en los resultados obtenidos).

Se eliminaron del estudio los pacientes que fallecieron tras la primera cirugía y aquellos en quienes no se realizó la adecuada valoración de APACHE II.

\section{RESULTADOS}

Se incluyeron en el estudio un total de 658 pacientes; de ellos, 248 (37.6\%) fueron del sexo femenino y 410 (62.3\%) del sexo masculino. La edad mínima fue de 18 años y la máxima de 89 , con una media de 51.87 años. En 395 pacientes se presentaban enfermedades concomitantes (60\%). A 380 (57.8\%) se les colocó condón y 278 (42.2\%) fueron manejados con otros métodos. De los 380 pacientes manejados con condón, 160 $(42.1 \%)$ fueron del sexo femenino y $220 \mathrm{del}$ sexo masculino (57.8\%).

En las pacientes del sexo femenino manejadas sin condón, el APACHE II bajó de 32 puntos a 22 puntos en un transcurso de siete días; en los pacientes masculinos, fue de 25 a 10 puntos en el mismo periodo. En quienes se colocó condón, la disminución de los valores de APACHE II fue de 26 a 10 puntos para el género femenino y de 22 a 4 puntos para el masculino.

En relación con el índice de Mannheim, se pudo observar que las pacientes de sexo femenino manejadas sin condón tuvieron una disminución de 35 a 25 puntos, mientras que los varones tuvieron una baja de 29 a 20 


\section{Cuadro III. Índice de peritonitis de Mannheim.}

\begin{tabular}{lc} 
Factor de riesgo & Valor \\
\hline Edad mayor a 50 años & 5 \\
Sexo femenino & 5 \\
Falla orgánica & 7 \\
Malignidad & 4 \\
Duración preoperatoria de la peritonitis mayor a 24 horas & 4 \\
Origen séptico no colónico & 4 \\
Peritonitis generalizada difusa & 6 \\
Exudado & $\mathrm{X}$ \\
Claro & 0 \\
Turbio o purulento & 6 \\
Fecal & 12 \\
\hline
\end{tabular}

Fuente: Malik AA, Wani KA, Dar LA, Wani MA, Wani RA, Parray FQ. Mannheim peritonitis index and APACHE II-prediction of outcome in patients with peritonitis. Ulus Travma Acil Cerrahi Derg. 2010; 16 (1): 27-32. puntos. Los valores del índice de peritonitis de Mannheim en pacientes en quienes se colocó el condón se redujeron de 31 a 15 puntos para el sexo masculino y de 31 a 14 puntos para el sexo femenino. Comparando el grupo donde se utilizó el condón con aquel donde no se utilizó, los valores bajaron de 31 a 15 y de 31 a 22 puntos del índice de peritonitis de Mannheim, respectivamente.

Los valores de APACHE II disminuyeron de 27 a 22 puntos en los pacientes sin condón y de 24 a 15 en aquellos con condón, una diferencia de siete puntos.

En los análisis estadísticos con chi-cuadrada $\left(\chi^{2}\right)$, se encontró que para el índice de peritonitis de Mannheim, en relación con los pacientes con condón, fue estadísticamente significativo, con un resultado de chi-cuadrada $\left(\chi^{2}\right)$ de 0.057 . No se halló significancia estadística en relación con comorbilidades o el sexo.

\section{DISCUSIÓN}

Con los resultados obtenidos hasta el momento, se puede inferir que en los pacientes manejados con el condón, el APACHE II tuvo una disminución más significativa que en aquellos abordados sin el mismo, aun cuando ésta no fue estadísticamente significativa.

También se observó que en relación con el índice de peritonitis de Mannheim, la reducción de los valores tuvo una significancia estadística, ya que se obtuvo un resultado de chi-cuadrada $\left(\chi^{2}\right)$ de 0.057 . Esto se traduce en que el condón utilizado en las fístulas intestinales podría ayudarnos a obtener un control de origen del foco séptico más rápido que cuando solamente realizamos aseos y lavados quirúrgicos, debido a una baja en la contaminación local y la respuesta inflamatoria, y al avance en el proceso cicatrizal.

Se encontró también que las pacientes del sexo femenino son las más beneficiadas con el uso del condón.

Se realizó una sistematización de la atención al paciente grave, donde los pacientes con Björck 3 y 4 son lo que se reciben ya con grados severos de desnutrición, sépticos y con alteraciones hepáticas por la nutrición parenteral por periodos prolongados. Implementar estrategias de cirugía de control de daños, nutrición enteral -aun con pequeños segmentos intestinales-y la utilización de fistuloclisis para aprovechar todo segmento intestinal disponible (la cual es una recomendación de las guías ESPEN 2017) nos ha otorgado la oportunidad de disminuir la mortalidad a menos del $30 \%$ (en la mayoría de las series es de más del $50 \%)$, reducir su estancia hospitalaria y poderlos manejar como ambulatorios hasta su reintervención, de seis meses a un año después de la falla intestinal derivada de una catástrofe abdominal.

Existen múltiples dispositivos para el manejo de pacientes con abdómenes Björck 3 y 4: sondas Foley, tubos endotraqueales, chupón o pezón artificial; estos fácilmente disfuncionan cuando el paciente empieza con dieta enteral o resultan ineficaces en panículos adiposos de más de cinco centímetros; los Penrose disfuncionan por acodamiento y fugan por inadecuada hermeticidad. Otros dispositivos más elaborados, como la "dona", la corona y el embudo de los sistemas de presión negativa, pueden dar los mismos resultados, pero con costos mucho más elevados (alrededor de un 300\%), y el paciente 
tendría que egresarse con el sistema de succión, sin tomar en cuenta que aún no está disponible en todas las unidades médicas, a diferencia del método del condón, que es accesible y de bajo costo.

Antes de la implementación de esta técnica en nuestro hospital, los pacientes en estas condiciones requerían estancias hospitalarias de seis meses a un año por el difícil control de la fuga intestinal, y desde la protocolización del manejo, la estancia hospitalaria ha disminuido significativamente: se han logrado incluso egresos hospitalarios en un promedio de tres o cuatro semanas, lo que repercute en los costos de hospitalización tanto en el medio público como en el privado.

El uso de esta técnica ha permitido lograr un control mucho más efectivo, sencillo y preciso de las fugas intestinales, así como el manejo de complicaciones estomales y un inicio temprano de dieta enteral (lo que mejora la supervivencia, evita complicaciones y disminuye costos hospitalarios); todo esto a un bajo costo y permitiendo mejorar la calidad de vida de los pacientes, para reintegrarse a su vida "cotidiana".

Sin embargo, ninguno de estos métodos sería necesario si se realizaran estomas a tiempo en un abdomen complicado o se evitara el uso de anastomosis "de riesgo", donde verdaderamente el único que se expone es el paciente.

Sólo falta romper paradigmas y perder el miedo al paciente grave.

\section{REFERENCIAS}

1. Maydón GH, Hernández VF, Esparza IR, Belmonte MC. Estomas intestinales: construcción y complicaciones. An Med (Mex). 2011; 56: 205-209.

2. Kann BR. Early stomal complications. Clin Colon Rectal Surg. 2008; 21: 23-30. doi: 10.1055/s-20081055318.

3. Velasco MM, Jiménez EF, Parajo CA. Estado actual de la prevención y tratamiento de las complicaciones de los estomas. Revisión de conjunto. Cir Esp. 2014; 92: 149-156.

4. Formijne $\mathrm{JH}$, Draaisma WA, Roskott AM, van Overbeeke AJ, Broeders IA, Consten EC. Early complications after stoma formation: a prospective cohort study in 100 patients with 1-year follow-up. Int J Colorectal Dis. 2012; 27: 1095-1099.

5. Trzciński R, Kresińska-Kaba T, Dziki A. Therapeutic management of stoma complications in selected patients. Pol Przegl Chir. 2013; 85: 496-504.
6. Husain SG, Cataldo TE. Late stomal complications. Clin Colon Rectal Surg. 2008; 21: 31-40.

7. Guía de práctica clínica. Tratamiento médiconutricional del paciente con estomas de eliminación de tubo digestivo. Consejo de Salubridad General. IMSS-646-13.

8. Malik AA, Wani KA, Dar LA, Wani MA, Wani RA, Parray FQ. Mannheim peritonitis index and APACHE II — prediction of outcome in patients with peritonitis. Ulus Travma Acil Cerrahi Derg. 2010; 16: $27-32$.

9. Meakins JL, Solomkin JS, Allo MD, Dellinger EP, Howard RJ, Simmons RL. A proposed classification of intraabdominal infections. Stratification of etiology and risk for future therapeutic trials. Arch Surg. 1984; 119: 1372-1378.

10. Knaus WA, Draper EA, Wagner DP, Zimmerman JE. APACHE II: a severity of disease classification system. Crit Care Med. 1985; 13: 818-829.

11. Billing A, Frölich D, Schildberg FW. Prediction of outcome using the Mannheim peritonitis index in 2003 patients. Peritonitis Study Group. Br J Surg. 1994; 81: 209-213.

12. Iñaguazo $D$, Astudillo M. Abdomen abierto en la sepsis intraabdominal severa. ¿Una indicación beneficiosa? Rev Chil Cir. 2009; 61: 294-300.

13. Smith BP, Adams RC, Doraiswamy VA, Negaraja V, Seamon MJ, Wisler J, et al. Review of abdominal damage control and open abdomens: focus on gastrointestinal complications. J Gastrointestin Liver Dis. 2010; 19: 425-435.

14. Park JJ, Del Pino A, Orsay CP, Nelson RL, Pearl RK, Cintron JR, et al. Stoma complications: the Cook County Hospital experience. Dis Colon Rectum. 1999; 42: 1575-1580.

15. Gorordo-Delsol L, Pérez-Nieto OR, Porras-Escorcia O, Altamirano-Arcos CA. Sepsis abdominal: fisiopatología, diagnóstico y tratamiento. Revista Mexicana de Cirugía del Aparato Digestivo. 2015; 4: 110-117.

16. Sarkut P, Dundar HZ, Tirnova I, Ozturk E, Yilmazlar T. Is stoma care effective in terms of morbidity in complicated ileostomies? Int J Gen Med. 2015; 8: 243-246.

17. Basilio A, López O, Olguín A, Delgadillo S. Abdomen abierto. Indicaciones técnicas y consecuencias. Trauma. 2005; 8: 32-36.

18. Asociación Mexicana de Cirugía General, AC. Comité de elaboración de guías de práctica clínica. Guía de práctica clínica: abdomen hostil. México D.F. Octubre del 2014.

19. Wainsteins De, Langer J. Abdomen abierto: indicaciones, manejo y cierre. En: Galindo F, et al. Enciclopedia de cirugía digestiva. Tomo I. 148: 1-21. www.sacd.org.ar

20. Badía TM, Llop TJ. Nutrición en el paciente quirúrgico. Cir Esp. 2014; 92: 377-378.

21. Llop J, Cobo S, Padulles A, Farran L, Jodar R, Badia MB. Soporte nutricional y factores de riesgo de aparición de fístulas enterocutáneas. Nutr Hosp. 2012; 27: 213-218. 


\section{LECTURAS RECOMENDADAS}

- Rogers VE. Managing preemie stomas: more than just the pouch. J Wound Ostomy Continence Nurs. 2003; 30: 100-110.

- Bastida IM, Medrano E, Terrero CJ, González MA. Mortalidad por peritonitis secundaria. UCl. Hospital VI Lenin 2000-2001. Correo Científico Médico de Holguin. 2002; 6.

- Cadena M, Vergara A, Solano J. Fístulas gastrointestinales en abdomen abierto. Revista de cirugía.

- Vega F, Millán JC, Castillo M, Larrinúa G, Zamudio I, Vázquez JC y cols. Tratamiento de la sepsis abdominal postraumática con técnica de abdomen abierto. Trauma. 20001; 4: 103-109.

\author{
Correspondencia: \\ Dr. Miguel Ángel Rivera Pérez \\ Calle del Bosque Núm. 2922, \\ Casa Blanca, 85134, \\ Cd. Obregón Sonora. \\ Tel. 6441732848 \\ E-mail: migueriv69a@hotmail.com
}

